\title{
Die Verteilung der Knorpeldegeneration an der menschlichen Patella in Beziehung zur individuellen subchondralen Mineralisierung
}

\author{
F. Eckstein, M. Miiller-Gerbl, R. Putz \\ Anatomische Anstalt München
}

\begin{abstract}
Zusanmenfassung
Nach Arbeiten von Palwels und Kummer kann die Verteilung der subchondralen Mineralisierung als Parameter der längerfristigen Beanspruchung in den Gelenken gelten. Ausgehend von eigenen Untersuchungen an der Patella zur Verteilung der subchondralen Knochendichte, wurden diese Befunde in der vorliegenden Arbeit den makroskopisch sichtbaren Knorpelschäden gegenübergestellt. Läsionen der lateralen Patellafacette sind Arealen hoher, Schäden der „odd facet" dagegen Arealen geringer subchondraler Mineralisierung zuzuordnen. Die Knorpelschäden der lateralen Facette werden als Folge einer hohen Dauerbeanspruchung und die Schäden der ,odd facet“ als Ausdruck einer generellen Unterforderung im medialen Gelenkbereich bei seltenen, kurzfristigen Spitzenbelastungen interpretiert.
\end{abstract}

\section{Distribution of Degenerative Changes of the Human Patella in Relation to the Individual Subchondral Mineralisation}

According to Pauswels and Kummer the distribution of the subchondral mineralisation can be taken as a measure of the long-term mechanical loading in the joints. Based on a previous study concerning the density distribution of the subchondral bone of the human patella, these results were compared in the following paper with the macroscopically visible cartilage lesions. Degenerative changes on the lateral facet of the patella coincide with regions of high bone mineralisation, those on the "odd facet" on the other hand with areas of low subchondral density. The cartilage lesions on the lateral facet are seen as being due to high and constant mechanical stress, those on the "odd facet" to short infrequent stress-peaks.

\section{Einleitung}

Das Femoropatellargelenk ist nicht nur der Ort der häufigsten und frühesten Knorpeldegeneration im menschlichen Körper überhaupt (Ficar u. Hungerford 1977), sondern kann nach diesen Autoren geradezu als Modell beim Studium von Knorpelschäden angeseben werden. Ficat (1973) bezeichnet es als ..observatoire ideal de l'arthrose". Allerdings konnte seit den ersten Beschreibungen femoropatellarer Knorpelschäden durch Büdinger (1906) keine Einigkeit über Verteilung, Progression oder Ätiologie dieser Läsionen erzielt werden. Eine Beteiligung mechanischer Faktoren an ihrer Entstehung ist unbestritten (Hehne 1983), zum exakten Wirkmechanismus bestehen jedoch recht unterschiedliche, zum Teil widersprüchliche Konzepte.

Mit der CT-Osteoabsorptiometrie (Müller-Gerbl u. Mitarb. 1989/1990) steht die Möglichkeit einer zerstörungsfreien, flächenhaften Darstellung der subchondralen Mineralisierung zur Verfügung, aus der auf die langfristige Beanspruchung in Gelenken rückge- schlossen werden kann (Pauwels 1965). In einer vorangehenden Arbeit (Eckstein u. Mitarb. 1992) konnten wir eine weitgehend konstante Verteilung der subchondralen Knochendichte nachweisen, die sich im Einklang mit modernen Konzepten zur Druckübertragung im Femoropatellargelenk befindet und als Ausdruck der individuellen mechanischen Beanspruchungssituation interpretiert werden kann.

Vor dem Hintergrund der Theorie von Radin u. Mitarb. (1970/1975/1978), die in einer Zunahme der Steifigkeit des subchondralen Knochens den Initialschritt zur Arthroseentwicklung sehen, erhebt sich die Frage. in welchem Zusammenhang die mittels CT-Osteoabsorptiometrie erhobenen Mineralisierungsmuster mit der Initiierung und Progression patellarer Knorpelschäden stehen.

Ziel der vorliegenden Untersuchung ist daher die makroskopische Dokumentation von Knorpelschäden an der menschlichen Patella und ihre Gegenüberstellung zur individuellen subchondralen Mineralisierung. 


\section{Material}

Wir untersuchten 30 Kniegelenkspräparate aus einum laufunden anatomischen Präparierkurs. welche in $4 \%$ iger Formalinlösung fixiert waren. Sie stellten eine Auswahl aus 120 Präparaten dar, wobei eine ausgewogene Altersverteilung mit einem möglicbst hoben Anteil von Präparaten jüngerer Individuen angestrebt wurde. Das erreichte Lebensalter betrug zwischen 47 und 90 Jahren. Fünfzehn Fälle mil einem Alter von weniger als 60 Jahren (Gruppe $\mathrm{J}=$ jung. Durchschnittsa)ter 53 Jahre) wurden 15 Präparaten von Menschen mit einem Lebensalter von über 60 Jahren (Gruppe $A=$ alt. Durchschnitsalter 73 Jahre) gegenübergestellt. Insgesamt sieben Präparate stammten von Frauen (Gruppe W) und wurden mit einer Auswahl von sieben Präparaten männlicher Individuen gleichen Lebensalters (Gruppe $M$ ) verglichen.

\section{Methoden}

Dokumentation der Knorpeldegeneration

Die Kniegelenke wurden im Präparicrkurs eröffnet, die patellare Gelenkfläche wurde freigelegt. Die makroskopisch sichtbare Knorpeldegeneration wurde in bezug auf ihre Ausdebnung sowie den Grad der Knorpelscbädigung in einer zweidimensionalen Gelenkflächenschablone, die nach Vermessung der Facettengrößen aller Kniescheiben angefertigt worden war, dokumentiert (Abb. 1). Wir stützten uns dabei auf eine bei der Arthroskopie übliche Klassifikation (Gschwend und Bischoffsberger 1971): Grad 1: blasige Auftreibung und Glanzverlust des Knorpels. Grad 2: Aufsplitterung und Zerklüftung. Grad 3: Eburnisierung des subchondralen Knochen. Die Knorpelschäden wurden anschlieBend je nach Grad mit Hilfe eines Graphikprogrammes (Studio 8) und eines Apple Macintosh Computers mit verschiedenen Graustufen belegt. In einem weiteren Schritt wurden Summarionsbilder für die Gesamtheit aller Kniescheiben suwie Untergruppen dargestellt, indem die Verteilungsmuster unter Zerlegung der Flächen in jeweils $50 \%$ der Bildpunkte sukzessive paarweise graphisch summiert wurden. Dabei zeigen die Grauwerte im Summationsbild den Schadensgrad. die Dichte der Bildpunkte die Häufigkeit im jeweiligen Areal an.

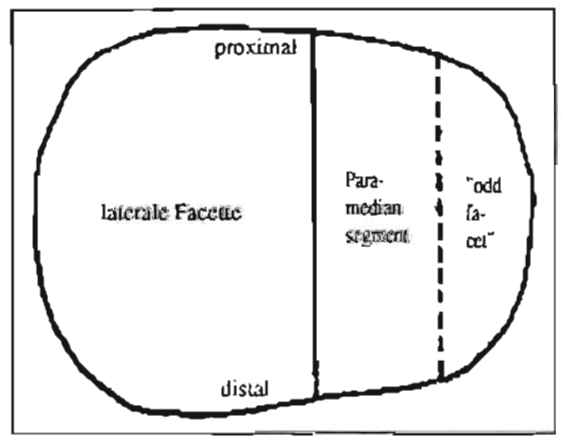

Abb. 1 Umrißschablone der $\mathrm{Ge}$ lenkliäche der $\mathrm{Pa}$ tella mit ihren drei Facetten, bestimmt aus 30 vermessenen Kniescheiben

\section{Bestimmung der subchondralen} Mineralisierung

Die Kniegelenke wurden in Extensionsstellung in einem Computertomographen (Siemens Delta Scan 50 FS 2) axial zwischen Tibiaplateau und oberem Patellapol geschichtet. An den im Abstand von $4 \mathrm{~mm}$ gewonnenen Schnitbildern mit einer überlappenden Schichtdicke von $8 \mathrm{~mm}$ wurden mittels ("T-Osteoabsorpliometrie (Müller-Gerbl u. Mitarb. 1989/1990) Isodensiten. d.h. Linien gleicher Hounsfielddichte von 400, 500. 600. 700 und 800 Hounsfield Units (HU) zur Darstellung gebracht. Die Dichte in $1.5 \mathrm{~mm}$ Tiefe unter der Gelenkfläche wurde auf die Gelenkfläche übertragen und aus den Schnittbildern die Verteilung in der Gelenkschablone rekonstruiert.

Die entstehenden sechs Bereiche von $<400 \mathrm{HL}$ ' bis $>800 \mathrm{HL}^{\prime}$ wurden im Computer mit genormten Grauwerten belegt. Anschließend erlolgte die Übereinanderschichtung diesur Muster zu Summationsbildern in einem Schwarz-Weiß-Mischprogramm. Für jede Durchschnitsverteilung wurden dic neu entstandenen Grauzwischenstufen in einem weiteren Schritt den jeweils nächstliegenden, ursprünglichen Graubereichen zugeordnet. um die Isodensiten im Summationsbild wiederherzustellen.

\section{Gegenüberstellung von \\ Knorpeldegeneration und subchondraler Mineralisierung}

Zum Vergleich der Verteilungen wurden an definierten Punkten der 30 Kniescheiben der Grad der Knorpelschädigung sowie der zugehörige Hounsfieldbereich abgelesen. Die Anzahl entsprechender Wertekombinationen wurde zunächst für alle 30 Patellae als prozentuale Verteilung der drei Knorpulschadensgrade in sieben Hounsfieldbereichen dargestellt und anschließend für die Gruppen J und A getrennt betrachtet, um einen Eindruck der Entwicklung der Knorpelschadensverteilung in den verschiedenen Hounsfieldbereichen unter Berücksichtigung des Lebensalters zu gewinnen.

\section{Ergebnisse}

\section{Knorpeldegeneration}

Knorpelschäden finden sich in allen Präparaten: Grad 1 an allen 30. Grad 2 an 29 und Grad 3 an 7 Präparaten. Die individuellen Verteilungsmuster zeigen eine große Variationsbreite. Häufig sind schwere Schäden (Grad 2 und 3) an der ,odd facet" (Randsegment) (Abb. 2a), am Sekundärfirst (Abb. 2b) und im Zentrum der lateralen Facette (Abb. 2c), Im Summationsbild (Abb. 3) wird deutlich. daß an der „odd facet" alle Grade der Knorpelschädigung gehäuft vorkommen und Schäden 2.13. Grades sich darüber hinaus oft am Sekundärfirst, im Zentrum der lateralen Facette und am distalen Patellarand finden.

Bei einem Vergleich der Gruppen $\mathbf{J}$ und A zeigt sich, daß in jüngerem Lebensalter die Schäden 


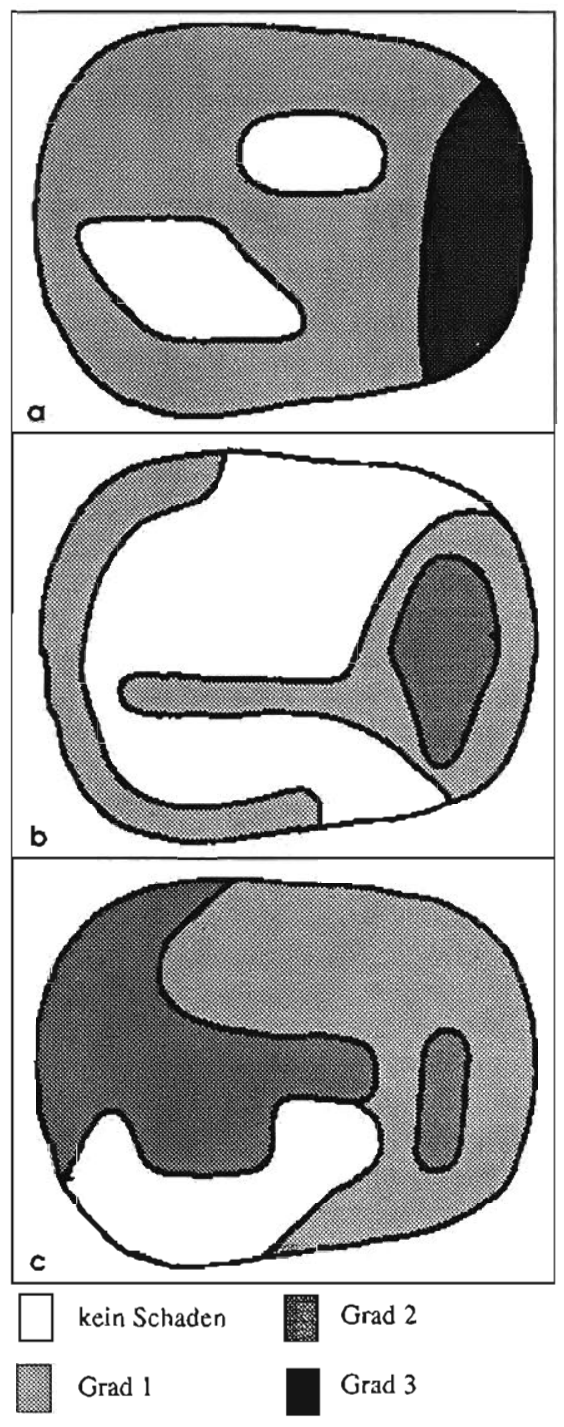

vornehmlich in der Patellaperipherie lokalisiert sind (Abb. 4a). In höherem Lebensalter nehmen schwere Schäden an Randsegment und Sekundärfirst zu und sind im lateralen Bereich zentral gelegen (Abb. 4b). Die Summationsbilder der Gruppen M und W weisen Unterschiede bei beiden Geschlechtern auf. Bei beiden Gruppen sind die Schäden an "odd facet" und Sekundärfist an sich sehr ausgeprägt. Während jedoch bei den Frauen die zentrale laterale Facette deutlich von Schäden 2./3. Grades in Mitleidenschaft gezogen ist (Abb. 5b), gehen bei den Männern die Schäden im Zentrum der lateralen Facette nicht über Grad 1 hinaus (Abb. 5a).

\section{Subchondrale Mineralisierung}

Die Maxima subchondraler Mineralisierung liegen konstant im proximalen Anteil der lateralen Facette und betragen zwischen 600 und $1100 \mathrm{HU}$. Die Dichtewerte nehmen zum Rand hin ab (Abb. 6), wobei die Verteilungsmuster bei einem Teil der Präparate konzentrisch konfiguriert sind, sich bei anderen dagegen inselförmige Dichteanstiege im Bereich des Sekundärfirstes beobachten lassen. Die „odd facet" ist subchondral nur gering mineralisiert.

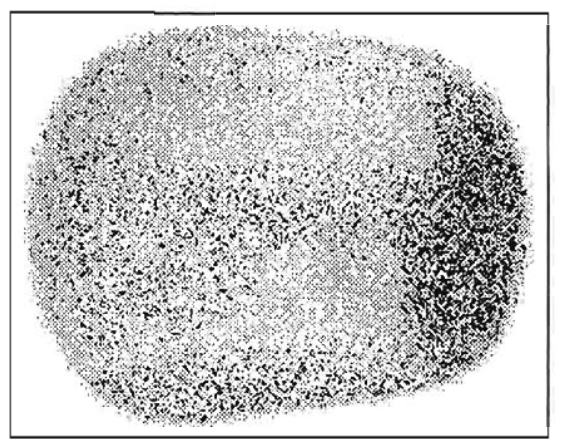

kein Knorpelschaden

Grad 1

Grad 2/3

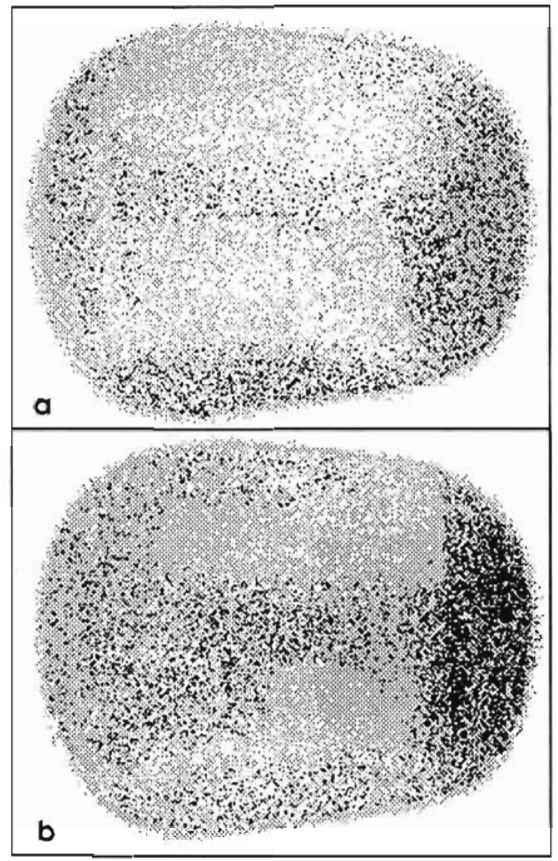

Abb. 4 Summationsbilder der Vertellung der Knorpeldegeneration a) Gruppe J (jung/ $n=151$ b) Gruppe A lalty $n=15$ )

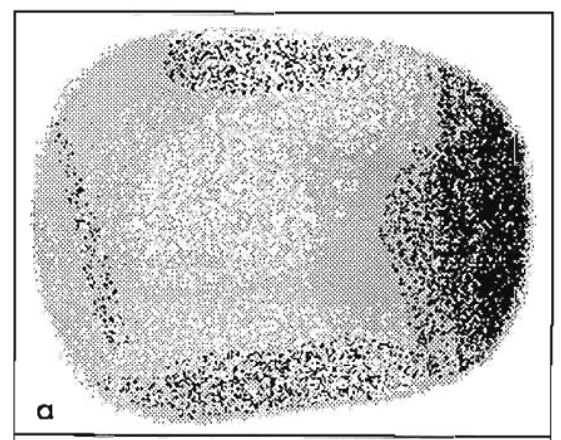

Abb. 5 Summatronsbilder der Verteilung der Knorpeldegeneration a) Gruppe $M$ (männlich/n=7) b) Gruppe W (weiblich $/ n=7$ ) 

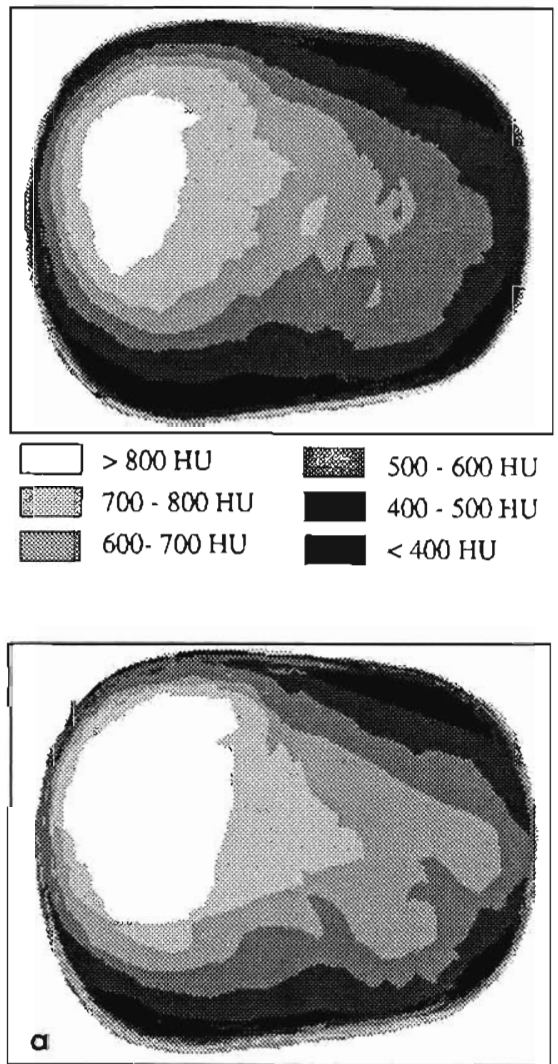

Abb. 7 Summatıonsbilder der Verteilung der subchondralen Mineralisierung

a) Gruppe J (jung/ $n=15$ )

b) Gruppe A lalt/ $n=15$ )
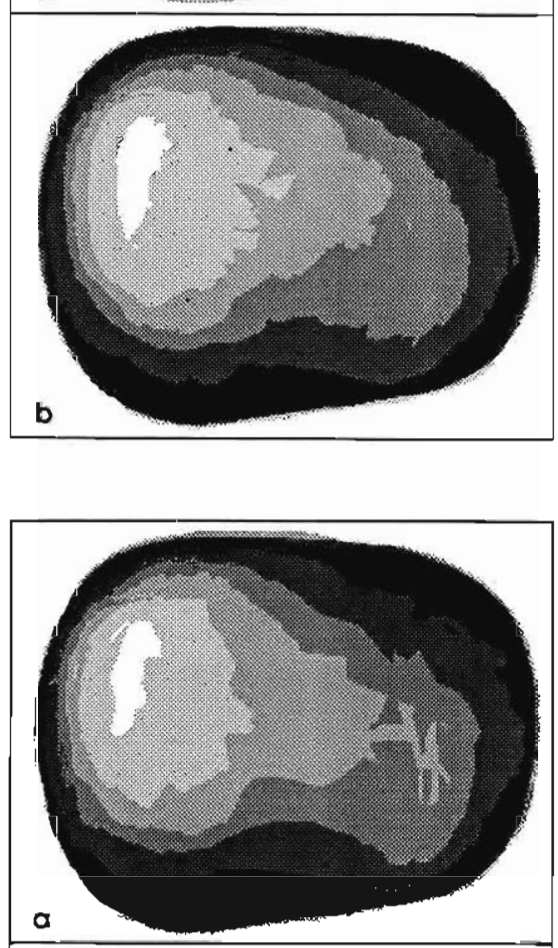

Abb. 8 Summationsbilder der Vertellung der subchondralen Mineralisierung

a) Gruppe $M$ (männlich/n = 7) b) Gruppe W iweiblich $/ n=7$ )
Bei der jüngeren Gruppe J findet sich eine größere Ausdehnung der höchsten Dichtestufe und eine Ausdehnung mittlerer Dichtebereiche weiter nach medial (Fig. 7a) als bei Gruppe A (Abb. 7b). Bei Gruppe $\mathrm{M}(\mathrm{Abb} .8 \mathrm{a})$ zeigt die höchste Dichtestufe eine geringfügig kleinere Ausdehnung als bei Gruppe W (Abb. 8b).

\section{Gegenüberstellung von \\ Knorpeldegeneration und subchondraler Mineralisierung}

Über Bereichen mittleren Mineralisierungsgrades (500-800 HU) ist der Knorpel prozentual am häufigsten ungeschädigt (30-39\%) und am seltensten von starken Knorpelschäden (Grad 2 und 3) betroffen $(7-13 \%)$ (Abb. 9). Sowoh] über Regionen geringer subchondraler Dichte $(<500 \mathrm{HU})$ wie sehr hoher Mineralisierung ( $>800 \mathrm{HU}$ ) ist der Knorpel seltener intakt (17$29 \%$ ) und häufiger stark geschädigt (16-33\%). Bei einem Vergleich der Durchschnittsverteilungen von Knorpelschäden (Abb. 3) und subchondraler Mineralisierung (Abb. 6) lassen sich die Knorpelschäden in den gering mineralisierten Bereichen der ,odd facet" zuordnen, diejenigen in hochmineralisierten Arealen dagegen der zentralen lateralen Facette. Dieser Zusammenhang läßt sich auch an Einzelbeispielen nachvollziehen, so stimmt bei Präparat Nr. 21 der Bereich zweitgradiger Schädigung (Abb. 10a) fast exakt mit dem Dichtebereich $>500 \mathrm{HU}$ (Abb. 10b) überein. Bei Präparat Nr. 24 liegt dagegen ein zweitgradiger Knorpelschaden des Randsegmentes (Abb. 11a) im Bereich < $400 \mathrm{HU}$ (Abb. 11b).

Vergleicht man die beiden Geschlechter (Abb. 5a/b und $8 \mathrm{a} / \mathrm{b}$ ), so fällt auf, daß die Knorpelschäden unterschiedlich gelegen sind. während bei der Verteilung der subchondralen Dichte weitgehende Übereinstimmung besteht. In der Gegenüberstellung von Gruppe J und A (Abb. 4a/b und 7a/b) zeigt sich, daß Knorpelschäden bei der älteren Vergleichsgruppe deutlich häufiger sind, während der subchondrale Knochen geringer mineralisiert ist.

Betrachtet man die prozentuale Verteilung der Knorpelqualität in den einzelnen Hounsfieldbereichen bei den verschiedenen Altersgruppen (Abb. 12), so ergibt sich zwischen Gruppe J und A eine höhere Differenz im Vorhandensein von intaktem Knorpel (Abb. $11 \mathrm{a})$ in den Arealen $>600 \mathrm{HU}(23-38 \%)$ als in den $\mathrm{Be}-$ reichen <600 HU (7-20\%). Bei Knorpelschäden 1. Grades liegt die Differenz höher in den Bereichen $>600 \mathrm{HU}$

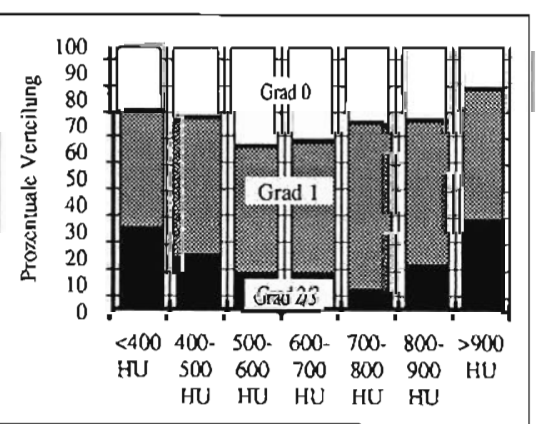

Abb. 9 Prozentuale Verteilung der Xnorpelschadensgrade in sieben Hounsfieldbereichen an allen Kniescheiben $(n=30)$. pelschaden Grad $0=$ kein Knor- 


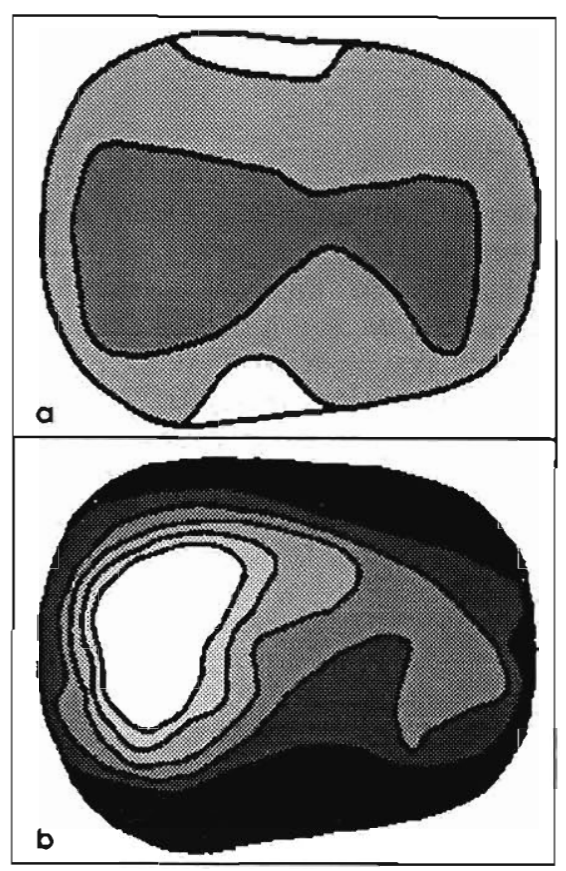

Abb. 10 Einzelbeispiel der Lokalisation eines schweren Knorpelschadens im Bereich hoher Mineralisierung (Präp. Nr. 21) a) Verteilung der Knorpeldegeneration

b) Verteilung der subchondralen Hounsfielddichte
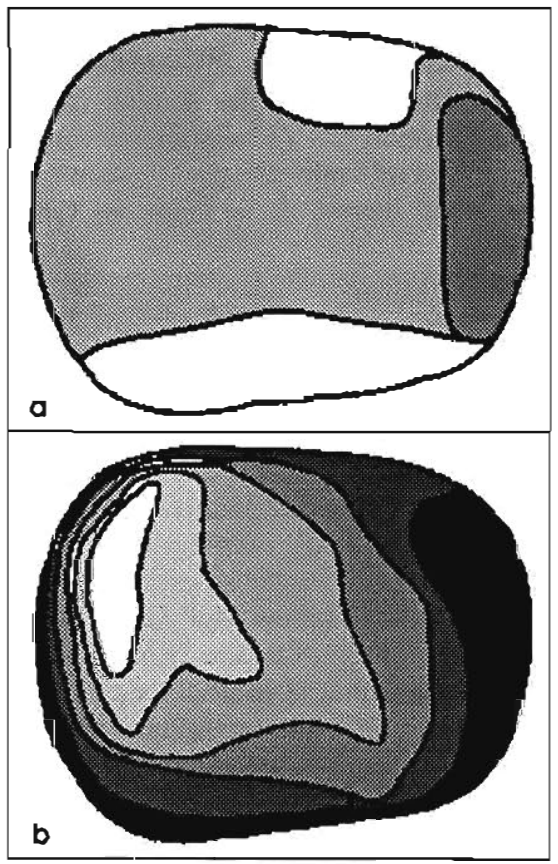

Abb. 11 Einzelbeispiel der Lokalisation eines schweren Knorpelschadens im Bereich geringer Mineralisierung (Präp. Nr. 24)

a) Verteilung der Knorpeldegenera tion

b) Verterlung der subchandralen Hounsfielddichte

(20-28\% gegenüber $3-10 \%$ bei $<600 \mathrm{HU})$ und bei Knorpelschäden 2. und 3. Grades in den Bereichen über 800 HU (18-33\% gegenüber $4-11 \%$ bei < $800 \mathrm{HU})$.

\section{Diskussion}

\section{Knorpeldegeneration}

Die Häufigkeit der gefundenen Knorpelläsionen entspricht der in der Literatur angegebenen extrem hohen Prävalenz patellarer Knorpelschäden (Meachim u. Emery 1974, Mirrovic u. Mitarb. 1987). Dabei sind die von uns beobachteten typischen Lokalisationen auch in der Literatur als solche beschrieben. so die

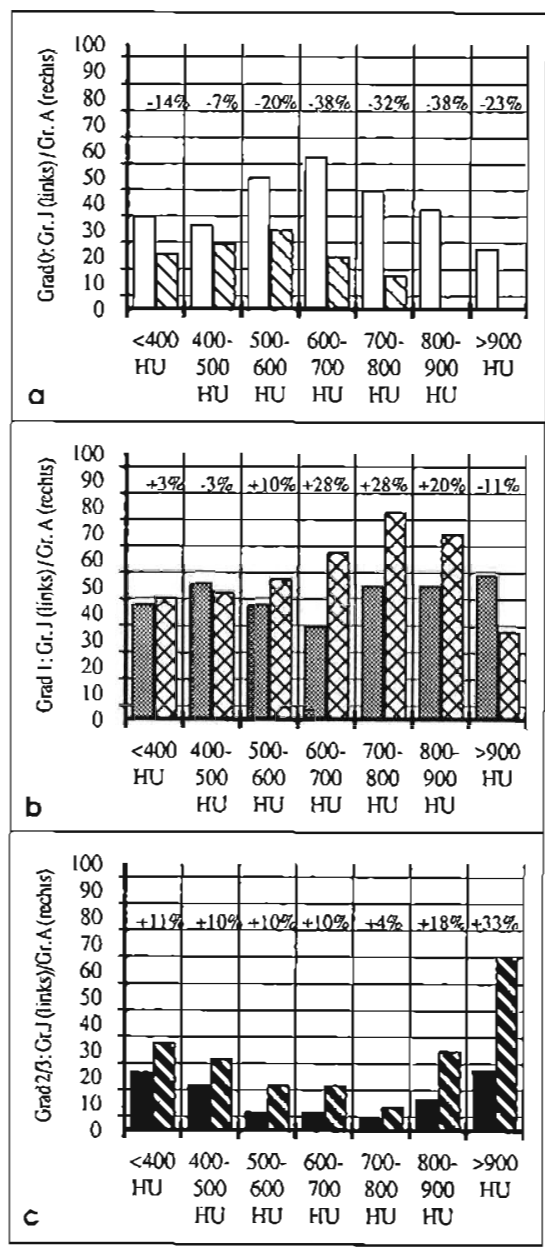

Abb. 12 Prozentuale Verteilung der Knorpelschadensgrade in sieben Hounsfieldbereichen vergleichend für Gruppe J (jung/ $n=15 /$ jeweils linke Säule) und Gruppe A lalt $/ n=15 /$ rechte Säule).

Prozentzahlen über den Säulen $=$ Differenz zwischen Gruppe J und A. a) Grad $0=$ kein Knorpelschaden

b) Grad 1 der Knorpelschädigung

c) Grad 2 und 3 der Knorpelschädigung

"Odd facet" von Goodfellow u. Mitarb. (1976) und Weh u. Lüer (1987), der Sekundärfirst von Hungerford u. Goodfellow (1975) und Hehne (1983), sowie die zentrale laterale Facette als ,zone critique“ der Knorpeldegeneration durch Ficat u. Mitarb. (1975) und Ficat u. Hungerford (1977).

Die Dominanz peripherer Schäden in jüngerem Lebensalter. wie sie bei Gruppe J zum Ausdruck kommt, erwähnen Emery u. Meachim (1973), das Auftreten zentraler Knorpelschäden erst in höherem Lebensalter Weh u. Lïer (1987). Wie Meachim u. Mitarb. (1977) und Mitrovic u. Mitarb. (1987) finden auch wir, daß Frauen von Knorpelschäden stärker betroffen sind als Männer, vor allem was zentrale Knorpelschäden betrifft. Der Grund für eine häufigere Knorpeldegeneration bei Frauen in diesem Gelenkbereich ist nach Meachim u. Mitarb. (1977) und Pedley und Meachim (1979) unbekannt.

\section{Subchondrale Mineralisierung}

Daß sich die gefundenen Verteilungsmuster subchondraler Mineralisierung als Ausdruck einer längerfristigen. durchschnittlichen Beanspruchungsverteilung im Femoropatellargelenk interpretieren lassen, konnten wir bereits in einer vorangehenden Arbeit (Eckstein u. Mitarb. 1992) zeigen. indem diese den Be- 
funden, die mittels Spannungsoptik und Spaltlinienanalyse (Tillmann u. Brade 1980) und Druckmeßfolie (Hehhe 1983) gewonnen worden waren, gegenúbergestellt wurden. Die Dichtemaxima an der proximalen lateralen Facette, welche bei allen Winkelgraden von 60 bis 140 Grad artikuliert, sehen wir als Folge der bei tiefer Kniebeugung ansteigenden Druckkräfte (Maquet 1976). Dje bei einem Teil der Präparate zu beobachtenden inselförmigen Dichteanstiege im Bereich des Sekundärfirstes interpretieren wir als Ausdruck einer nahezu punktförmigen Auflage bei 120 Grad Beugewinkel (Hehne 1983).

Die geringe Mineralisierung der „odd facet" führen wir auf ihre druckmäßig zwar hohe (Maquel 1976/Hehne 1983), aber zeitlich wohl eher seltene Beanspruchung (geringe relative Verweildauer der Resultierenden) bei 140 Grad Kniebeugewinkel zurück. Die etwas größere Ausdehnung von Bereichen höherer und mittlerer Mineralisierung bei jüngeren Individuen kann als Ausdruck einer für diese Gruppe vermuteten größeren Beugeaktivität angesehen werden.

\section{Gegenüberstellung von \\ Knorpeldegeneration und subchondraler Mineralisierung}

Knorpelschäden in Bereichen sehr hoher Mineralisierung, wie sie z. B. auch am Hüftgelenk dokumentiert sind (Pauwels 1968, Tillmann 1978), können als Folge einer hohen Dauerbeanspruchung gesehen werden. wie sie angesichts der konstanten Lage subchondraler Dichtemaxima an der proximalen lateralen Facette zu vermuten ist. $\mathrm{Zu}$ ähnlichen Ergebnissen kommen aufgrund klinischer und röntgenologischer Untersuchungen auch Ficat u. Mitarb. (1975) sowie Ficat u. Hungerford (1977), die ein Hyperpressionssyndrom der lateralen $\mathrm{Pa}$ tellafacette als eigenständiges Krankheitsbild herausarbeiten. Die Knorpelschäden am Sekundärfirst könnten dagegen durch dessen hohe Beanspruchung bei der nahezu punktförmigen Auflage bei 120 Grad Beugewinkel bedingt sein. Dies wird durch die bei einem Teil der Präparate beobachteten inselförmigen Dichteanstiege in diesem Bereich nahegelegt und wird auch von Hungerford u. Goodfellow (1975) und von Hehne (1983) hervorgehoben.

Die hohe Tendenz zur Knorpeldegeneration in niedrigmineralisierten Bereichen betrifft nach unseren Befunden in erster Linie die „odd facet". Sie artikuliert bei einem Beugewinkel von 140 Grad (Hehne 1983) und ist dabei hohen Anpreßdrücken unterworfen (Maquet 1976). Ihre geringe subchondrale Mineralisierung und die daraus abzuleitende Vermutung einer geringen relativen Verweildauer der Resultierenden in diesem Gelenkbereich steht in Übereinstimmung mit der Aussage von Goodfellow u. Mitarb. (1976), daß die starke Schädigung dieser Facette mit ihrer seltenen Beanspruchung im Alltagsleben von Europäern zusammenhängt. Eine Erklärung dieser Schäden mit einer chronischen Minderernährung des Knorpels, die auf hohen medialen Knorpeldicken beruhen soll (Paar 1978, Morscher und Dick 1980), lehnen wir allerdings ab, da wir in einer vorangehenden Arbeit (Eckstein u. Mitarb. 1992) zeigen konnten. daß in den meisten Fällen höhere Knorpeldik- ken lateral zu finden sind und dort oft keine derartigen Knorpelschäden vorkommen.

Wir vermuten vielmehr, daß seltene Belastungsspitzen. wie sie an der „odd facet" auftreten, eine für das „Überleben“ des Gelenkknorpels ungünstige mechanische Umgebung schaffen und daß in Arealen mittleren Mineralisierungsgrades die häufigere Beanspruchung einen protektiven Faktor vor Knorpeldegeneration darstellt. Eine solche „Trainierbarkeit" des Gelenkknorpels könnte durch eine Dickenanpassung des Gelenkknorpels an die Beanspruchungsverteilung im Femoropatellargelenk (Putz u. Mitarb. 1987, Eckstein u. Mitarb. 1992) gegeben sein. Diese Interpretation steht auch in Einklang mit Befunden von Hoaglund u. Mitarb. (1973) und Marar u. Mitarb. (1975), die die im Verhältnis zu Europäern bei Asiaten weit selteneren Schäden an der Patella in Zusammenhang mit deren Sitzgewohnheiten in tiefer Hockstellung (squatting), d. h. mit einer zeitlich häufigeren Beanspruchung der „odd facet", bringen.

Die unterschiedlichen Verteilungsmuster patellarer Knorpelschäden bei beiden Geschlechtern lassen sich auf der Basis subchondraler Dichtedifferenzen nicht erklären. da die Mineralisierungsmuster bei beiden Gruppen kaum Unterschiede ausweisen. Ob hierbei andere, z. B. hormonelle Faktoren eine Rolle spielen, oder ob es sich bei diesen Differenzen um Zufallsbefunde aufgrund relativ geringer Präparatezahlen $(n=7)$ handelt. läßt sich im Rahmen unserer Studie nicht beantworten.

Die Theorie von Radin u. Mitarb. (1970/ 1975/1978), die eine Zunahme subchondraler Knochensteifigkeit als Initialschritt der Arthroseentstehung interpretieren, können wir angesichts unserer Befunde an sich nicht ausschließen, da durch die von uns erhobenen Zustandsbilder potentielle Initialveränderungen nicht unbedingt erfaßt werden. Sie kann durch unsere Befunde aber auch nicht bestätigt werden, da trotz ganz unterschiedlicher Schädigungsmuster in individuellen Fällen und beim Vergleich der beiden Geschlechter kaum Verteilungsunterschiede bei der subchondralen Mineralisierung zu beobachten sind. Dagegen spricht unserer Ansicht des weiteren, daß bei der älteren Vergleichsgruppe, bei der die Knorpelschäden deutlich häufiger zu beobachten sind, die subchondrale Dichte eher niedriger liegt. Es muß darüber hinaus die Einschränkung gemacht werden. daß von der subchondralen CT-Dichte nicht direkt auf die mechanischen Eigenschaften des subchondralen Knochens geschlossen werden kann. Da wir jedoch große Ähnlichkeiten der von uns gefundenen Mineralisierungsmuster mit einer Festigkeitsverteilung des subchondralen Patellaknochens nach Townsend $\mathrm{u}$. Mitarb. (1976) beobachten und Benizen u. Mitarb. (1987) am Tibiaplateau bereits bohe Korrelationen von CT-Dichte und physikalisch-mechanischen Parametern nachweisen konnten, halten wir vergleichende Aussagen für erlaubt.

Nach Abernethy u. Mitarb. (1978) sollen steile Gradienten der Knochensteifigkeit an der Initiierung von Knorpelschäden ursächlich beteiligt sein, da der Knorpel bei Druckbeanspruchungen lokalen Scherkräften unterworfen wird. Diese Meinung ist mit unseren 
Befunden vereinbar, da aus obengenannten Überlegungen die steilen Dichteabfälle im Randbereich der Patella, wo der Knorpel sehr früh zur Degeneration neigt, solche Gradienten der Knochensteifigkeit nahelegen. Die von Radin u. Mitarb. (1978) formulierte und von Pedley und Meachim (1979) überprüfte Hypothese der Bedeutung einer hohen subchondralen Mineralisierung für die Progression von Knorpelschäden trifft auch auf unser Untersuchungsgut zu, da im Vergleich der jüngeren und älteren Gruppe die Knorpelschäden in den hochmineralisierten Bereichen ( $>600 \mathrm{HU}$ ) stärker zunehmen und die zweit- und drittgradigen Läsionen vor allem in den Arealen $>800 \mathrm{HU}$ voranschreiten.

Unsere Befunde zeigen, daß patellare Knorpelschäden in gleicher Weise wie arthrotische Veränderungen in anderen Gelenken aus einem Mißverhältnis von Beanspruchung und Belastbarkeit des Gelenkknorpels entstehen (Pauwels 1968, Tillmann 1978), wobei wir einer hohen Dauerbeanspruchung im lateralen Bereich und medial einer generellen Unterforderung des Knorpels mit seltenen, kurzfristigen Belastungsspitzen eine besondere Bedeutung beimessen. Eine gleichmäßig intermittierende Beanspruchung scheint dagegen die besten „Überlebenschancen“ für den Gelenkknorpel zu gewährleisten.

Die subchondrale Dichte können wir unseren Ergebnissen nach nicht, so wie Radin u. Mitarb. (1970/1975) dies nahelegen, quasi als einen „Hauptakteur" im Schauspiel der arthrotischen Degeneration interpretieren. wohl aber als eine "Schaubühne“, die die Bedingungen der ablaufenden Handlung festlegt.

\section{Literatur}

Abernethy, P. J., P. R. Townsend, R. M. Rose, E. L. Radim: Is chondromalacia patellae a separate clinical entity? J. Bone Joint Surg. 60B (1978) 205-210

Benrzen, S. M.. I. Hvid, J. Jorgensen: Mechanical strength of tibial trabecular bone evaluated by X-ray computed tomography. J. Biomech. 20 (1987) 743-752

Büdinger, K.: Über Ablösung von Gelenkıeilen und verwandte Prozesse. Deutsche Zisch.r. F. Chir. 84 (1906) 311-365

Eckstein, F., M. Müller-Gerbl. R. Putz: Distribution of the subchondral bone density and cartilage thickness in the human patella. J. Anat. 180 (1992) $425-433$

Emery, I. H. G. Meachint: Surface morphology and topography of patello-femoral cartilage fibrillation in Liverpool necropsies. J. Anat. 116 (1973) $103-120$

Ficat, P.: Les deséquilibres rotuliens de l'hyperpression à l'arthrose. Masson, Paris (1973)

Ficat, $P$. C. Ficat, A. Bailleux: Syndrome d'hyperpression externe de la rotule (SHPE). Son intéret pour la connaissance de l'arthrose. Rev. Chir. Orthop. 61 (1975) 39-59

Ficat, P., D. S. Hungerford: Disorders of the patellofemoral joint. Masson. Paris (1977)

Goodfellow. J., D. S. Hungerford. C. Woods: Patello-femoral joint mechanics and pathology. 2. Chondromalacia patellae. J. Bone Joint Surg. 58B (1976) 291-299
Hehne, H. J.: Das Patellofemoralgelenk. Enke, Stuttgart (1983)

Hoaglund, F. T. A. C. M. C. Yau, W. L. Wong: Osteoarthritis of the hip and other joints in Southern Chinese in Hong Kong. Incidence and relited factors. J. Bone Joint Surg. 55A (1973) 545-557

Hungerford, D. S., J. W. Goodfellow: Femoropatellare Kontakuzonen und ihre Beziehung zur Chondromalazie. Z. Orthop. 113 (1975) 784786

Maquel, P. G. J.: Biomechanics of the knee. Springer. Berlin-Heidelberg-New York (1976)

Marar, B. C. M. C. Orth, V. K. Pillay: Chondromalacia of the patella in Chinese. J. Bone Joint Surg. 57A (1975) 342-345

Meachim. G., I. H. Emery: Quantitative aspects of patellofemoral cartilage fibriltation in Liverpool necropsies. Ann. Rheum. Dis. 33 (1974) 3947

Meachim, G., G. Bentley. R. Baker: Effect of age on thickness of adult patellar articular cartilage. Ann. Rheurn. Dis. 36 (1977) 563-568

Mirrovic, D., A. Stankovic, O. Borda-Iriurte, M. Uzan, M. Quintero, A. Ryckewaert: Résultats de l'examen autopsique des cartilages des genoux chez 120 sujets décédés en milieu hospitalier 1 . Articulation fémoro-patellaire. Rev. Rheum. Mal. Osteoarsic. 54 (1987) 15-21

Morscher, E. W. Dick: Die sagittale Patellaosteotomie bei Chondromalacia palellae. Orthop. Praxis 8 (1980) 692-695

Müller-Gerbl. M., R. Puiz, N. Hodapp. E. Schulte, B. Wimmer: Computed tomography-osteoabsorptiometry for assessing the density distribution of subchondral bone as a measure of long term mechanical adaptation in individual joints. Skeletal Radiol. 18 (1989) 507-512

Müller-Gerbl, M., R. Pluz, N. Hodapp, E. Schulke. B. Wimmer. Die Darstellung der subchondralen Dichtemuster mittels der CT-Osteoabsorptiometrie (CT-OAM) zur Beurteilung der indiviauellen Gelenkbeanspruchung am Lebenden. Z. Ortliop. 128 (1990) 128-133

Paar, O.: Zur Problematík der Chondropathia patellae, Unfallheilkunde 81 (1978) 694-697

Pauwels, F.: Gesammelte Abhandlungen zur Biomechanik des Bewegungsapparates. Springer, Berlin-Heidelberg-New York 1965

Pauwels, F.: Der Platz der Osteotomie in der operativen Behandlung der Coxarthrose. Triangle 8 (1968) 196-210

Pedley, R. B. G. Meachim: Topograplical variation in patellar subarticular calcified tissue density. J. Anat. 128 (1979) 737-745

Pulz, R., M. Miller-Gerbl, E. Schulhe, B. Wimmer: Verteilung der Knorpeldichte und der Mineralisierung im Kniegelenk. In: Der alloplastische Ersatz des Kniegelenkes. Hrsg. von Refior, H. J., M. H. Hackenbroch. C. J. Wirth, Georg Thieme Verlag, Stuttgart-New York (1987)

Radin, E. L., 1. L. Paul, M. Lowy: A comparison of the dynamic force transmitting properties of subchondral bone and articular cartilage. J. Bone Joint Surg. 52A (1970) 444-456

Radin, E. L., I. Paul, R. M. Rose: Mecluanical factors in the aetiology of osteoarthrosis. Ann. Rheum. Dis. (Suppl.) 34 (1975) 132-133

Radin, E. L., P. J. Abernethy, P. M. Townsend, R. M. Rose: The role of bone changes in the degeneration of articular cartilage in osteoarthrosis. Acta Orthop. Beig. 44 (1978) 55-63

Tillmann, B.: Funkionelle Morphologie und Beanspruchung der Gelenke. Verh. Anal. Ges. 72 (1978) 47-63

Tillmann, B., H. Brade: Morphologische und biomechanische Untersuchungen an der Facies articularis patel)ae. Orthop. Praxis 6 (1980) 462467

Townsend, P. R., R. E. Miegel, R. M. Rose, P. Raux, E. L. Radin: Structure and function of the human patella: The role of cancellous bone. J. Biomed. Mater. Res. Symposium 7 (1976) 605-611

Weh, L., C. Lïer: Plica medialis, Patellaform und Chondromalazie. Z. Orhop. 125 (1987) 54-62

Dr. F. Eckstein. Dr. M. Müller-Gerbl, Prof. Dr. R. Putz

A natomische Anstalt

Ludwig-Maximilian-Universität München

Pettenkoferstr. II

D-80336 München 